\title{
L'implication des parties prenantes dans la démarche évaluative : facteurs de succès et leçons à retenir
}

\author{
Isabelle Bourgeois \\ École nationale d'administration publique \\ Marthe Hurteau \\ Université du Québec à Montréal
}

Résumé : Les trois articles de cette section thématique abordent le rôle et la participation des parties prenantes dans la démarche évaluative selon des angles différents. Nous reprenons dans ce texte les points principaux des trois articles, afin d'identifier les facteurs de succès quion y retrouve et d'en tirer des leçons. Pour ce faire, nous nous inspirons des "Principes visant à guider les approches collaboratives en évaluation", identifiés par Shulha, Whitmore, Cousins, Gilbert et Al Hudib (2016).

Mots clés : évaluation collaborative, parties prenantes

\begin{abstract}
Our cross-cutting overview of the three papers that make up this thematic segment shows that each of the papers addresses the issue of stakeholder involvement quite differently from the others. We focus here on the key messages from each of these papers in order to highlight success factors and lessons learned for stakeholder participation in evaluation. Success factors related to collaborative approaches to evaluation are also presented throughout the analysis, based on the "Principles guiding collaborative approaches to evaluation" recently published by Shulha, Whitmore, Cousins, Gilbert et Al Hudib (2016).
\end{abstract}

Keywords: collaborative evaluation, stakeholders

\section{INTRODUCTION}

Le colloque de l'ACFAS que nous avons organisé en 2016 et dont sont issus les textes de cette section thématique visait à explorer l'implication et la contribution des parties prenantes à lévaluation de programme. Cet enjeu d'actualité a déjà fait l'objet de nombreuses publications scientifiques et continue à intéresser les chercheurs et les évaluateurs souhaitant peaufiner les théories, concepts et pratiques associés à l'intégration des parties prenantes, soit les intervenants, gestionnaires, bénéficiaires, organisations et la société civile à la démarche évaluative. Au départ,

Correspondance à l'auteur : Isabelle Bourgeois, École nationale d’administration publique; isabelle.bourgeois@enap.ca

(c) 2018 Canadian Journal of Program Evaluation / La Revue canadienne d'évaluation de programme 33.2 (Fall / automne), 236-246 doi: 10.3138/cjpe.42205 
les écrits dans le domaine portaient surtout sur les modes et types de participation des parties prenantes afin de favoriser l'utilisation des résultats dévaluation. Aujourd'hui, les études sur le sujet sont beaucoup plus nuancées et portent sur des thèmes différents, dont les compétences personnelles et culturelles, la participation dans divers secteurs d'intervention, le renforcement des capacités en évaluation et l'apprentissage organisationnel. Les trois textes présentés dans cette section thématique s'inscrivent dans cette ligne de pensée en décrivant les études déjà publiées de façon accessible et organisée (Marchand), les démarches collaboratives entreprises lors de l'évaluation de deux programmes d'intervention psychosociale (Dubeau, Bérubé, Lafantaisie, Coutu et Turcotte) et la contribution des compétences personnelles de lévaluateur en offrant un nouvel éclairage, soit celui de la sagesse pratique (Houle, Hurteau et Marchand).

Le présent texte vise à intégrer les constats principaux des articles qui composent cette section thématique en soulevant les questions suivantes :

1. Pourquoi impliquer les parties prenantes dans le cadre d'une évaluation?

2. Quels sont les facteurs de succès d'une évaluation collaborative qui implique les parties prenantes?

3. Quelles leçons peut-on tirer de telles expériences?

Nous reprenons ces questions, ainsi que certains éléments tirés des trois articles afin dorienter notre réflexion au sujet des enjeux soulevés par les auteurs.

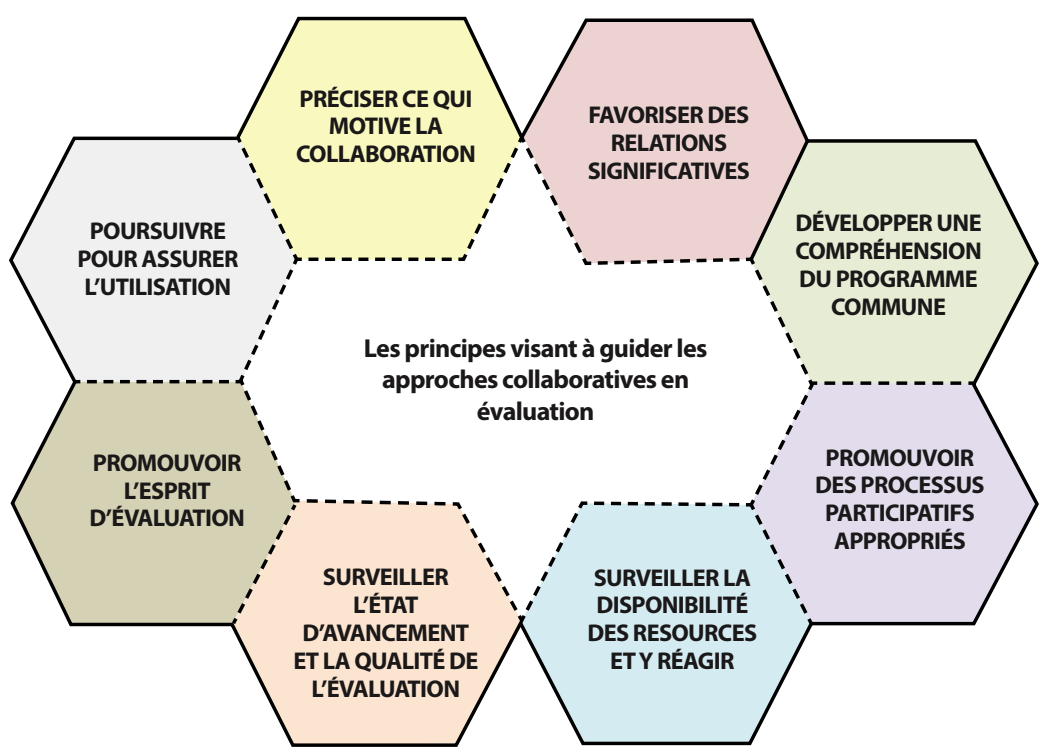

Figure 1. Principes visant à guider les approches collaboratives en évaluation (Shulha, Whitmore, Cousins, Gilbert et Al Hudib, 2016). 
Pour ce faire, nous nous basons sur les Principes visant à guider les approches collaboratives en évaluation, élaborés par Shulha, Whitmore, Cousins, Gilbert et Al Hudib (2016). Ces principes, validés lors d'une étude pluriannuelle impliquant au-delà de 320 évaluateurs, visent à établir les fondements d'une pratique collaborative en évaluation. Ils ont été conçus pour orienter les décisions, choix et actions des évaluateurs tout au long de la démarche évaluative. Tout comme Marchand, ces auteurs ne cherchent pas à camper leur analyse dans une approche évaluative particulière, ce qui rend les principes qu'ils proposent appropriés pour toute forme dévaluation - participative, collaborative, ou transformative. L'intégration de ces principes, présentés ci-dessous, nous permet d'approfondir notre réflexion et d'en tirer des leçons pratiques pour l'implication des parties prenantes.

\section{POURQUOI IMPLIQUER LES PARTIES PRENANTES DANS LE CADRE D'UNE ÉVALUATION?}

Marchand dresse en quelque sorte la table dans son introduction en affirmant que : "Bien qu'elle puisse entraîner son lot de tensions (Hurteau, 2009) et que tous n'y adhèrent pas sans réserve (Daigneault et Jacob, 2012), nombreux sont les praticiens et théoriciens qui prônent l'importance de la participation des parties prenantes au processus évaluatif de par les avantages qu'elle procure, d’où la naissance et l'effervescence des multiples approches inclusives. » Plusieurs raisons seront évoquées par la suite pour justifier leur inclusion, que ce soit l'évolution disciplinaire, la reconnaissance de leur pertinence, la production d'un jugement crédible et l'utilisation des résultats et recommandations découlant de l'évaluation. Chacune de ces raisons sera développée dans les points suivants, mais auparavant, mentionnons que, peu importe les raisons choisies pour impliquer les parties prenantes, il est nécessaire, selon Shulha et coll. (2016) de "préciser ce qui motive la collaboration ", l'un des principes visant les approches collaboratives. Par exemple, Dubeau et ses collègues ont sélectionné une approche collaborative lors de l'évaluation de Relais-Pères en raison du grand nombre de partenaires impliqués dans la prestation de l'intervention et de sa complexité.

\section{L'évolution disciplinaire}

Selon Marchand, l'implication des parties prenantes au sein d'une démarche évaluative suit le cours naturel de lévolution de la discipline de l'évaluation de programme. En effet, le passage d'un paradigme positiviste à des paradigmes plus pragmatistes et constructivistes a permis l'intégration graduelle des acteurs. Certaines approches évaluatives, telles que l'évaluation axée sur l'utilisation, lévaluation participative pratique et lévaluation développementale, cherchent à impliquer un large éventail de parties prenantes afin de rendre lévaluation plus utile aux décideurs et aux principaux intervenants. D’autres approches évaluatives, telles que lévaluation participative transformationnelle, souhaitent plutôt intégrer certains acteurs dans une perspective de renforcement des capacités et d'émancipation (Cousins et Whitmore, 1998). 


\section{La reconnaissance de leur pertinence}

De nos jours, l'implication des parties prenantes peut prendre plusieurs formes, allant de la simple consultation des intervenants-clés lors de lélaboration du cadre dévaluation à des formats beaucoup plus participatifs où les acteurs sont impliqués à toutes les phases et dans tous les aspects de la démarche évaluative (Daigneault et Jacob, 2012). Selon Marchand, leur sélection ainsi que l'attribution de leurs tâches et rôles au sein de la démarche évaluative dépend de facteurs individuels, tels que le rôle que joue la partie concernée par rapport au programme à évaluer, ses caractéristiques sociodémographiques, ses compétences interpersonnelles et son pouvoir d'influence. L'importance de ces facteurs, et de leur influence sur la sélection des parties prenantes, nest pas à négliger afin que lévaluateur puisse s'entourer des personnes pertinentes au bon déroulement des activités. Dans certains cas, comme le suggèrent Dubeau et ses collègues, les parties prenantes prendront plutôt la forme dorganismes, comme illustré par le cas de lévaluation du programme Relais-Pères. Cette réalité complexifie la démarche puisque les individus représentant ces organismes peuvent changer au fil de la démarche évaluative, et leur sélection est souvent établie par lorganisme concerné. Les évaluateurs doivent ainsi faire preuve d’adaptabilité.

\section{Favoriser la production d'un jugement crédible}

L'implication des parties prenantes est aussi associée à la production d'un jugement crédible. Comme le soutiennent Houle et ses collaboratrices, la démarche évaluative doit favoriser le dialogue et les échanges entre lévaluateur et les parties prenantes afin d'assurer le respect des valeurs de ces dernières et ainsi favoriser l'acceptabilité des résultats générés. Selon ces auteurs, une démarche d'évaluation qui n’intègre pas les parties prenantes risque, en fin de compte, de donner lieu à un rejet ou à une contestation des résultats de l'évaluation. Ainsi, en impliquant les parties prenantes dans un processus de co-construction du jugement, les évaluateurs assurent la légitimité, la crédibilité et l'acceptabilité de lévaluation.

\section{Favoriser l'utilisation des résultats et des recommandations découlant de l'évaluation}

L’une des raisons les plus fréquemment évoquées pour justifier l'inclusion des parties prenantes dans la démarche évaluative est celle de l'utilisation de l'évaluation. Cette problématique, qui fait l'objet détudes et de questionnements depuis les débuts de lévaluation, perdure encore aujourd'hui (Johnson et coll., 2009). Elle est reflétée dans l'un des principes énoncés par Shulha et coll. (2016), soit " poursuivre pour assurer l'utilisation ». Selon Marchand, l'objectif ultime de lévaluation collaborative est souvent l'utilisation des résultats de l'évaluation. Ce principe vise la reconnaissance de cet objectif et précise davantage le rôle de lévaluateur et des parties prenantes, qui doivent assurer la diffusion et l'utilisation des résultats de lévaluation. Les évaluateurs, avant tout, souhaitent que leur travail ait un sens, une valeur, aux yeux des acteurs organisationnels. En d'autres mots, ils souhaitent que 
leur travail mène à l'amélioration des programmes, des services et des interventions, à une meilleure prise de décisions fondée sur les données probantes, et à une plus grande justice sociale. En partie, l'implication des parties prenantes permet au minimum la construction "d'une compréhension du programme commune » (Shulha et coll., 2016) : lors des étapes préliminaires d'une évaluation, les évaluateurs et les parties prenantes doivent s'entendre sur l'objet même de l'évaluation. Pour ce faire, l'élaboration d'un modèle logique, tel que décrit par Dubeau et ses collègues, permet de décrire l'intervention et les résultats visés de manière concise et claire, et ainsi, de développer une compréhension uniforme du programme.

Dans le même ordre d'idées, l'implication des parties prenantes tout au long de la démarche évaluative, selon Johnson et coll. (2009), permet d'assurer une meilleure utilisation de l'évaluation, qu'elle soit instrumentale (application directe des résultats de lévaluation), conceptuelle (l'évaluation mène à une meilleure compréhension du programme et de son contexte) ou du processus (les acteurs impliqués dans une démarche évaluative font l’acquisition de nouvelles compétences et appliquent ces compétences à l'ensemble de leurs activités organisationnelles). Selon Shulha et coll. (2016), l'utilisation du processus vise à " promouvoir l'esprit dévaluation » au-delà des activités d'évaluation comme telles. Comme nous l'avons constaté dans le texte de Marchand, l'implication des parties prenantes a souvent comme objectif de renforcer les capacités organisationnelles en évaluation dans une visée émancipatoire ou d'apprentissage organisationnel. On peut même, dans certains cas, parler d'un changement de la culture organisationnelle qui n'est possible que lorsque les membres de l'organisation adhèrent à une vision commune de lévaluation et reconnaissent son pouvoir d'influence sur l'atteinte des objectifs organisationnels. Dubeau et ses collègues font aussi référence à la création des conditions permettant de vivre une expérience positive liée à lévaluation. La participation active des parties prenantes et la transparence quant aux relations de pouvoir feront en sorte, selon ces auteurs, de promouvoir l'esprit dévaluation en la rendant plus agréable et stimulante.

Cependant, cette implication demeure fragile et Houle et ses collaboratrices insistent sur les qualités personnelles essentielles de lévaluateur, dont la sagesse pratique, pour gérer efficacement la démarche dans son ensemble ainsi que les éventuels écueils qui peuvent surgir en cours de route et compromettre son issue.

\section{QUELS SONT LES FACTEURS DE SUCCÈS D'UNE ÉVALUATION COLLABORATIVE QUI IMPLIQUE LES PARTIES PRENANTES?}

\section{Bien comprendre les contextes de l'intervention et de l'évaluation}

Selon Dubeau et ses collègues, les contextes multiples qui entourent l'intervention et son évaluation exercent une influence importante sur les choix de lévaluateur quant à l'inclusion des parties prenantes. Les caractéristiques globales politiques, géographiques et économiques, en plus du secteur d'intervention et du champ disciplinaire qui y est associé, influencent entre autres les choix méthodologiques 
de l'évaluateur, et, ainsi, la mesure dans laquelle certaines parties prenantes seront impliquées dans la démarche évaluative. Les caractéristiques plus spécifiques de l'organisation (ex. : sa grandeur, son chiffre d'affaires, sa clientèle cible) auront aussi une influence marquée sur l'implication des parties prenantes. Lévaluateur doit donc étudier ces contextes avant de prendre des décisions relatives à l'implication des parties prenantes, afin de s'assurer que l'approche évaluative retenue ainsi que les méthodes de recherche reflètent les contraintes associées au contexte et soient réalisables.

\section{Planifier les interactions avec les parties prenantes}

Les interactions avec les parties prenantes tout au long de la démarche évaluative doivent être planifiées et structurantes. Cette planification doit tenir compte, entre autres, des activités d'évaluation et des informations recherchées et rejoint le principe de "promouvoir des processus participatifs appropriés » énoncés par Shulha et coll. (2016). Ce principe favorise une réflexion soutenue sur les responsabilités de chacun au processus d'évaluation et comment les contributions de tous les membres du groupe seront mises à profit. Comme souligné par Marchand, ce principe exige une réflexion sur le contrôle du processus décisionnel, la sélection et diversité des parties prenantes ainsi que leur degré de participation. Cela représente un défi pour Dubeau et ses collègues, qui soulèvent la « cohabitation d'acteurs aux valeurs, aux cultures organisationnelles, aux expertises et aux intérêts différents » comme étant une contrainte importante et pour laquelle la mise en place des processus participatifs appropriés est essentielle. Une planification détaillée est particulièrement importante lorsque l'évaluation est réalisée par plusieurs organismes travaillant en partenariat, comme dans les deux cas présentés par l'équipe de Dubeau, ou encore, lorsque l'intervention est réalisée par plusieurs organismes travaillant en partenariat. Des ententes formelles ou informelles quant à la fréquence des rencontres, aux participants, et aux thèmes qui seront abordés peuvent se révéler très utiles lorsque l'évaluation est en cours. Une bonne planification permet aussi d'alléger la lourdeur du processus en identifiant les balises temporelles du projet. Dans des cas moins complexes, où les acteurs sont impliqués de façon moins intensive, une stratégie de communication permet à l'évaluateur d'anticiper les besoins informationnels des divers types de parties prenantes et de s'assurer que les informations leur seront communiquées au moment opportun.

Selon Shulha et coll. (2016), l'évaluateur doit aussi s'assurer de " surveiller l'état d'avancement et la qualité de l'évaluation ». Dans le cadre d'une évaluation impliquant les parties prenantes, ce principe revêt une importance particulière, surtout lorsque les évaluateurs ne contrôlent pas entièrement la démarche. Leur rôle, dans ce cas, est d'assurer la qualité des produits d’évaluation générés par les parties prenantes ainsi que le maintien des normes professionnelles de l'évaluation. Dubeau et ses collègues identifient cette responsabilité importante comme condition gagnante dans le cadre de l'évaluation de Relais-Pères, où la création d'un Comité de pilotage a permis d'effectuer une surveillance de la démarche évaluative 
tout en permettant aux participants d'échanger sur les pratiques en cours. Houle, Hurteau et Marchand décrivent aussi la sagesse pratique comme étant constituée des compétences personnelles des évaluateurs, dont celle de pouvoir apporter rapidement les ajustements requis au protocole et à la méthodologie en fonction des circonstances, sans pour autant amoindrir la qualité de la démarche.

\section{Engager les ressources humaines et financières nécessaires à la démarche}

Les évaluations qui impliquent des parties prenantes prennent généralement plus de temps et de ressources financières que les évaluations qui se font à distance de l'intervention étudiée. Le succès de ces évaluations repose donc en partie sur la disponibilité des ressources nécessaires, qu’elles soient humaines, financières, ou matérielles. L'un des principes de Shulha et coll. (2016) soulève l'importance de "surveiller la disponibilité des ressources et y réagir ». Par exemple, Dubeau et ses collègues font référence à des ententes de partenariats qui comprennent, entre autres, des ressources matérielles (salles de réunion) et des ressources humaines (assistants de recherche). Sans ces appuis, la démarche collaborative risque de ne pas être possible. De plus, ces auteurs identifient le temps comme une « denrée rare [...] un ingrédient essentiel pour construire la coopération entre les partenaires et développer une vision ainsi qu'une compréhension communes de la situation et de l'action à entreprendre dans le respect des philosophies de chacun » (p. 209). En plus de s'assurer que les ressources suffisantes seront disponibles, il s'agit aussi, selon ce principe, de surveiller leur utilisation tout au long de lévaluation et d’ajuster les plans en conséquence. Les ressources financières et humaines, ainsi que le temps, doivent être continuellement évaluées et réaffectées aux diverses activités dévaluation. Le temps des évaluateurs et des parties prenantes, surtout dans le cas d'une évaluation externe, doit être comptabilisé et planifié dès le départ, en tenant compte des imprévus possibles, afin d'éviter des situations difficiles en cours de route.

\section{Favoriser des relations significatives et porteuses}

En plus des compétences techniques ou méthodologiques nécessaires à toute évaluation, Houle, Hurteau et Marchand identifient des compétences personnelles, notamment la sagesse pratique, qui permettront d'assurer le bon déroulement de lévaluation et de "favoriser des relations significatives » (Shulha et coll., 2016). Il est essentiel, au cours d'une évaluation collaborative, d'assurer une relation de travail de qualité entre les évaluateurs et les parties prenantes ainsi que le respect, la confiance et la transparence au cours des échanges, mais surtout de saisir les situations conflictuelles qui émergent en cours de route et dont l'issue peut remettre en question la démarche. Marchand, ainsi que Houle et ses collègues, décrivent les « compétences relationnelles » qui font partie des compétences personnelles de l'évaluateur et qui favorisent la production d'un jugement crédible. On pense notamment à une ouverture sur les autres, à l'empathie et à la création d'un climat de confiance et de collaboration et, finalement, à la sagesse pratique. On pense aussi aux compétences communicationnelles, qui permettent à lévaluateur de 
développer un argumentaire solide et à transmettre un message clair aux parties prenantes. Les auteurs réfèrent à ces compétences comme étant nécessaires à la production d'un jugement acceptable aux yeux des parties prenantes. En d'autres mots, lévaluateur doit posséder les compétences nécessaires pour reconnaitre les particularités d'une situation et pour choisir le scénario optimal afin de bien faire les choses. Comme le mentionne House (2015) : « practical wisdom is not about establishing rules and following them. It's about if, when, and how to apply the rules. » (p. 90). Dubeau et ses collègues décrivent le « capital relationnel», qui réfère à la convergence des intérêts des partenaires, ainsi que des expériences de collaboration antérieures positives. Le maintien de relations significatives contribue, entre autres, à la définition et au partage des rôles et responsabilités, le travail conjoint, le partage des savoirs, et l'engagement continu des partenaires face au projet dévaluation (Dubeau et collègues). Houle, Hurteau et Marchand appuient aussi ce principe en mettant en évidence l'importance d'une démarche évaluative co-construite avec les parties prenantes en tant que garantie du maintien de leur adhésion et de leur acceptabilité des résultats.

\section{QUELLES LEÇONS PEUT-ON TIRER DE CETTE ANALYSE?}

Les contributions conceptuelles et empiriques des auteurs du segment thématique nous permettent de tirer certaines conclusions par rapport à l'implication des parties prenantes en évaluation. Nous retenons, notamment, les quatre points suivants, lesquels illustrent aussi les principes énoncés par Shulha et coll. (2016) :

1. Les approches évaluatives impliquant les parties prenantes doivent tenir compte des ressources financières et humaines nécessaires au partage, à la collaboration, et à la co-construction de lévaluation.

2. La sélection des parties prenantes doit être réfléchie de façon à favoriser la pleine participation des individus disposant des qualités et ressources nécessaires au succès de lévaluation. Ces choix dépendront en grande partie du contexte dans lequel lévaluation aura lieu ainsi que de ses objectifs.

3. Les interactions entre l'évaluateur et les parties prenantes doivent être planifiées et réfléchies afin dêtre productives et soutenues tout au long de l'évaluation. Cependant, il faut reconnaitre qu’une démarche évaluative est un processus dynamique, tout autant que les relations entre les parties prenantes et l'évaluateur. Lémergence d'événements imprévisibles place lévaluateur à une croisée de chemin et les décisions qu'il prendra feront la différence entre le succès ou l'échec de la démarche. Dans ce sens, il est souhaitable que les évaluateurs et les parties prenantes impliquées dans une démarche dévaluation aient des compétences techniques et personnelles favorisant la production d'un jugement crédible.

4. L'implication des parties prenantes dans une démarche dévaluation doit avoir des objectifs bien précis : le renforcement des capacités en évalu- 
ation des organismes partenaires, par exemple, ou encore l'utilisation instrumentale des résultats de l'évaluation.

\section{CONCLUSION}

Les trois articles de cette section thématique présentent différentes manières d'aborder le rôle des parties prenantes dans la démarche évaluative. Initialement, l'implication des parties prenantes a été envisagée comme source essentielle d'information dans le cadre d'une démarche évaluative ainsi que comme levier nécessaire à l'utilisation des résultats qui en découlent (Alkin, 2013). Cette thématique toujours en évolution a fait l'objet de plusieurs synthèses, dont le texte de Marchand, qui propose de revisiter le thème de l'implication des parties prenantes en effectuant un survol de la littérature existante à ce jour. Larticle définit certains concepts essentiels et présente les différentes approches, modalités et processus favorisant leur sélection et leur participation. Dubeau, Turcotte, Bérubé, Lafantaisie et Coutu décrivent comment certaines parties prenantes organisationnelles, ou partenaires, ont été impliquées dans deux projets de recherche évaluative et documentent les conditions gagnantes favorisant cette participation. Il sagit, selon ces auteurs, 1) de miser sur un capital relationnel pour la création de liens de confiance, 2) de tenir compte des contextes global et local dans lesquels s'inscrivent l'intervention et l'évaluation et 3) d'associer les acteurs de la recherche aux différentes étapes de développement d'un projet. Dans ce sens, ils notent que de répondre rapidement aux besoins des parties prenantes facilite lémergence de ces liens. De plus, les auteurs précisent la nécessité de la mise en place de structures adéquates qui assurent un fonctionnement souple et flexible contribuant à une gouvernance efficace.

Si on prend en considération l'ensemble des conditions gagnantes pour assurer le succès de la démarche évaluative, et plus spécifiquement l'aspect relationnel qui y prend une place importante, il devient évident que l'évaluateur doit maîtriser un éventail imposant de compétences, et plus spécifiquement des dispositions personnelles. Hurteau et Houle (2008) avaient effectué le même constat, mais à l'époque, les auteurs n'avaient pas été en mesure de pleinement développer ce volet, faute de référent adéquat. House (2015, p.88) leur offre cette opportunité en introduisant le concept de sagesse pratique qu'il définit comme «... doing the right thing in the special circumstances of performing the job ». Pour lui, la sagesse pratique ne consiste pas tant à appliquer des règles, comme à établir quand et comment les appliquer de façon à assurer le succès de l'entreprise. Cela a amené les auteurs de l'article à réinterpréter leur étude initiale dans le but d'intégrer des données qui étaient demeurées « orphelines», pour ne pas dire « tabou ». Effectivement, la sagesse pratique trouve sa manifestation dans le cadre d'événements imprévisibles, qui peuvent apparaître anodins aux premiers abords mais dont la gestion est déterminante. Cette option requiert une analyse constante du contexte afin d'effectuer les ajustements requis, des interventions empreintes d'empathie et de respect envers les personnes impliquées. Le jugement qui en découle gagne à être validé auprès de pairs. Les auteurs précisent que cela demande une certaine 
vigilance de la part de lévaluateur pour éviter de manquer d’empathie ou de faire preuve d'un " excès de confiance ", attribuable à l'impression de reconnaitre la situation en l'associant à des situations antérieures familières. Un tel glissement pourrait constituer un facteur important d'échec.

Le présent article a effectué une synthèse des articles à la lumière des Principes visant à guider les approches collaboratives en évaluation, élaborés par Shulha et coll. (2016). Il ressort de l'analyse de ces articles une expression concrète des principes de manière concrète, ce qui suggère une certaine cohérence sur le plan des résultats des études et des principes. Ce constat nous permet d’alléguer que les approches évaluatives qui intègrent les parties prenantes partagent certains objectifs communs, tels que l'utilisation des résultats de lévaluation, la justice sociale, et l'apprentissage organisationnel.

\section{RÉFÉRENCES}

Alkin, M.C. (éd.). (2013). Evaluation roots: A wider perspective of theorists' views and influences. Los Angeles: Sage.

Cousins, J.B. et Whitmore, E. (1998). Framing participatory evaluation [Understanding and practicing participatory evaluation]. New Directions for Evaluation, 80, 5-23.

Daigneault, P.-M. et Jacob, S. (2012). Conceptualiser et mesurer la participation à lévaluation. Dans V. Ridde et C. Dagenais (éds.), Approches et pratiques en évaluation de programmes (pp. 233-254). Montréal: Presses de l’Université de Montréal.

House, E.R. (2015). Evaluating: Values, biases, and practical wisdom. Charlotte, NC: Information Age Publishing Inc.

Hurteau, M. (2009). Évaluation des programmes : ses visées? qui la pilote? et qui y participe? Dans L. Mottier Lopez et M. Crahay (éds.), Évaluations en tension : entre la régulation des apprentissages et le pilotage des systèmes (pp. 113-126), Neuville La Louve: De Boeck.

Hurteau, M. et Houle, S. (2008). Plus qu'une introduction, un énoncé. Dans M. Hurteau, S. Houle et F. Guillemette (éds.), Lévaluation de programme axée sur le jugement credible (pp. 1-12). Presses de l'Université du Québec: Québec.

Johnson, K., Greenseid, L.O., Toal, S.A., King, J.A., Lawrenz, F. et Volkov, B. (2009). Research on evaluation use: A review of the empirical literature from 1986 to 2005. American Journal of Evaluation, 30(3), 377-410. https://doi.org/10.1177/1098214009341660

Shulha, L., Whitmore, E., Cousins, J.B., Gilbert, N. et Al Hudib, H. (2016). Introducing evidence-based principles to guide collaborative approaches to evaluation: Results of an empirical process. American Journal of Evaluation, 37(2), 193-215. https://doi. org/10.1177/1098214015615230

\section{PRÉSENTATION D'AUTEUR}

Isabelle Bourgeois, Ph. D., est professeure agrégée à l'École nationale d'administration publique (ENAP). Ses travaux de recherche portent principalement sur le renforcement des capacités organisationnelles en évaluation. Elle est la rédactrice de la Revue canadienne d'évaluation de programme. 
Marthe Hurteau, Ph. D., est professeure titulaire au département d'éducation et pédagogie, Faculté des sciences de léducation de l'Université du Québec à Montréal (UQAM). Elle détient le titre d'évaluatrice accréditée décerné par la Société canadienne dévaluation (SCE). 